\title{
Blue loops of intermediate mass stars
}

\section{CNO cycles and blue loops}

\author{
H. Y. Xu ${ }^{1}$ and $\mathrm{Y} . \mathrm{Li}^{1,2}$ \\ 1 National Astronomical Observatories/Yunnan Observatory, PO Box 110, 650011 Kunming, PR China \\ 2 Joint Laboratory for Optical Astronomy, Chinese Academy of Sciences, PR China
}

Received 12 May 2003 / Accepted 19 December 2003

\begin{abstract}
We investigate the effects of the $\mathrm{CNO}$ cycles on the formation of the blue loop for intermediate mass stars of solarlike metallicity. By use of two ways to treat the CNO cycles, we find that the models adopting the CNO bi-cycles (CNO models) develop extensive blue loops while those only considering the $\mathrm{CN}$ cycle ( $\mathrm{CN}$ models) do not. We compare the properties of the $\mathrm{CN}$ and $\mathrm{CNO}$ models to explore the triggering mechanism of the blue loop. We notice that during the blue loop the increase of luminosity is determined by the nuclear energy production in the stellar core while the increase of effective temperature measures how far the stellar envelope can expand for a given luminosity at its base. We find that the stellar envelope obeys the virial theorem to a very high accuracy. Thus in a convection-dominated envelope, the extra heat from its base will lead to more developed convective motion and the decrease of temperature, and the star evolves up along the RGB. However in a radiationdominated envelope, the increase of luminosity requires the increase of temperature to enlarge the radiation transfer efficiency, and the star develops a blue loop. We introduce an envelope convective ratio $\eta$, which is defined by the envelope convection mass divided by the total envelope mass, to measure the development of convection in the stellar envelope. It is found that the critical value, $\eta_{\text {crit }}$, for a star to develop a blue loop is between 0.3 and 0.45 , and $\eta_{\text {crit }}$ shows a weak dependence on the stellar mass. On the other hand, we find that the enhancement of the energy production rate in the H-burning shell is responsible for the increase of the stellar luminosity during the blue loop phase. The increasing central He-burning rate increases the temperature of the H-burning shell, while the movement of the H-burning shell along the chemical profile enhances its energy production efficiency. We find that higher ${ }^{14} \mathrm{~N}$ abundance in the H-burning shell plays a crucial role in the formation of the blue loop for the CNO models. It makes the H-burning shell not only move quickly toward the lower temperature region in the pre-loop phase to decrease the stellar luminosity and $\eta$, but also place the hydrogen discontinuity closer to the shell source to increase the stellar luminosity during the blue loop phase by adding higher abundant hydrogen into the H-burning shell. We find that overshooting from the convective core makes the abundance discontinuity layer displace farther from the stellar core, which makes the energy production of the H-burning shell less efficient and the blue loop more difficult to be formed. The opacity enhancement of the OPAL over the LAOL leads to stronger convective motion in the stellar envelope and thus makes the blue loop more difficult to form.
\end{abstract}

Key words. stars: evolution - stars: interiors - stars: Hertzsprung-Russell (HR) and C-M diagrams

\section{Introduction}

During the core He-burning phase, intermediate mass stars may evolve from the red-giant branch (RGB) to the blue-giant region and return in the HR diagram (HRD), and this phenomenon is called blue loops. The blue loop was first found by Hayashi et al. (1962), and Kippenhahn and his co-workers made extensive investigation of the properties of the blue loop (Hofmeister et al. 1964; Kippenhahn et al. 1965; Kippenhahn $\&$ Weigert 1967). On the observational side, many stars are found in the blue-giant branch (Mermilliod 1981; Schmidt 1984; Evans 1993), and this fact requires the stars to spend

Send offprint requests to: $\mathrm{H}$. Y. Xu, e-mail: xuhuayin@sohu.com considerable time in that part of the HRD. The only explanation for these blue-giant stars is that they are in the blue loop phase, for the first crossing of the stars from blue to red over this area of the HRD is too short for us to see them (Chiosi \& Maeder 1986). Current evolutionary models are in good agreement with the observations, but the triggering of the blue loop is still a puzzling problem.

The formation mechanism of the blue loop seems a controversial problem that has been discussed for many years and given rise to different views. Kippenhahn \& Weigert (1990) considered the formation of the blue loop as a result of the $\mathrm{H}$ burning shell moving outward through an increasing hydrogen abundance profile. They introduced an effective core potential 
(Lauterborn et al. 1971a), $\varphi=h M_{\mathrm{C}} / R_{\mathrm{C}}$, to measure the distance of a model from the Hayashi line on the HRD, and found that a blue loop occurred when $\varphi<\varphi_{\text {cr }}$. They assumed that $h$ was proportional to the mass fraction of helium just above the shell source in the chemical profile. As the outward movement of the shell source decreased the value of $h$, a blue loop would appear when $\varphi<\varphi_{\mathrm{cr}}$.

Based on the "mirror reflection principle" that was encountered in various stages of stellar evolution, Fricke \& Strittmatter (1972) ascribed the blue loop evolution to the variation of the stellar core. They found that the helium core complied with the virial theorem. In the early core He-burning stage, the mean molecular weight increased rapidly and the stellar core had to expand correspondingly. This effect cooled the H-burning shell located just exterior to the helium core, and the stellar envelope would contract to keep the stellar luminosity almost unchanged by increasing the density of the shell source. Thus the star evolved blueward. In the late stage of the core He-burning, on the other hand, the helium abundance was fairly low and the stellar core contracted to keep the core luminosity nearly constant. Then the stellar envelope correspondingly expanded to cause the star to evolve redward.

Iben \& Renzini (1984) and Renzini et al. (1992) regarded the evolution of stellar models from blue to red in the post main sequence phase and from red to blue in the blue loop phase as a thermal non-equilibrium response of the stellar envelope to the variation of the core luminosity, which was a variation of the so-called gravothermal hysteresis cycle (Lynden-Bell \& Wood 1968; Fujimoto \& Iben 1991; Iben 1993). If the core luminosity was higher than the stellar luminosity, the envelope was heated and expanded, which resulted in the decrease of temperature in the envelope. As the envelope obeyed the virial theorem, some of the core luminosity was absorbed by the envelope and the rest emerged on the stellar surface. The increase of opacity blocked the heat transfer through the envelope, and more and more heat was absorbed to drive the envelope expansion farther and farther. This feedback led the stellar envelope to be thermally unstable, and pushed the star quickly from the main sequence to the red-giant branch. Based on similar arguments, the reverse process made the star move blueward and closed the cycle if the core luminosity was lower than the stellar luminosity. However this unstable cycle occurs on the thermal time scale, and Renzini et al. (1992) considered the interaction between the shell source and the stellar envelope to account for the blue loop evolution that was mostly carried out on the nuclear time scale. They suggested that a blue loop would be developed if $L<L_{\text {loop }}$ when the star dropped down to the bottom of the red-giant branch.

Another plausible opinion on the triggering of the blue loop is related to the hydrogen discontinuity that marked the former base of the homogeneous convective envelope when the envelope convection penetrated most inwardly (Robertson 1972; Stothers \& Chin 1973; Schlesinger 1977; Stothers \& Chin 1991a).

The blue loop depends sensitively on many physical factors adopted in the stellar models. For example, outward overshooting from the convective core during the main sequence phase can significantly suppress the subsequent development of the blue loop (Matraka et al. 1982; Huang \& Weigert 1983; Bertelli et al. 1985), while the inward overshooting from the base of the convective envelope counteracts this effect and facilitates the development of the blue loop (Alongi et al. 1991; Stothers \& Chin 1991a). The use of the Schwarzschild criterion or Ledoux criterion also makes different blue loops (El Eid 1995). Increasing the rate of reaction ${ }^{12} \mathrm{C}(\alpha, \gamma){ }^{16} \mathrm{O}$ can extend the blue loop on the HRD (Iben 1967; Bertelli et al. 1985; Brunish \& Becker 1990). The decrease of metal abundance, which leads to the decrease of opacity, sometimes produces more extended blue loops but sometimes not (Castellani et al. 1990; Alongi et al. 1991; Schaller et al. 1992; Bono et al. 2000). Mass loss in the red-giant phase is a strong effect to suppress the blue loop (Lauterborn et al. 1971a; Lauterborn \& Siquig 1974; Bertelli et al. 1985; Bono et al. 2000), but massive stars sometimes develop extended blue loops when strong mass loss is taken into account (Bertelli et al. 1985; Salasnich et al. 1999). It is well known that the shape and extension of the blue loop are different with different opacity tables (Fricke et al. 1971; Carson \& Stothers 1976; Bressan et al. 1993; Stothers \& Chin 1968, 1991b, 1993, 1994; Cassisi et al. 1994). Stellar rotation can stretch the blue loop (Kippenhahn et al. 1970), while the reverse opinion exists, too (Endal \& Sofia 1976). There are so many physical processes affecting the blue loop that a detailed understanding of their influence and mutual interactions is far from obtained. In the present paper, we investigate the development of the blue loop by use of two different methods to treat the CNO cycles, to see the role of energy generation on the triggering of the blue loop during the evolution of intermediate mass stars.

The physical input adopted in our model calculations is discussed in Sect. 2. The properties of the evolution models are discussed in Sect. 3, placing the emphasis on the effect of different treatments of the CNO cycles on the development of the blue loop. In Sect. 4 we investigate the structure of the stellar models during the blue loop phase, and attempt to clarify the formation mechanism of the blue loops. Our main conclusions and discussions are given in Sect. 5.

\section{Physical assumptions and computation methods}

We used $\mathrm{C}_{12}, \mathrm{~N}_{14}$, and $\mathrm{O}_{16}$ to denote the number density and $X_{\mathrm{C}}, X_{\mathrm{N}}$, and $X_{\mathrm{O}}$ to denote the mass abundance of ${ }^{12} \mathrm{C},{ }^{14} \mathrm{~N}$, and ${ }^{16} \mathrm{O}$, respectively. The $\mathrm{CNO}$ cycles, which provide most of the nuclear energy for intermediate mass stars in the main sequence and in the shell H-burning stage, could be approximated with enough accuracy by the $\mathrm{CN}$ and $\mathrm{NO}$ bi-cycle. The energy produced by the CNO cycles is mainly controlled by the abundance of ${ }^{1} \mathrm{H}$ and ${ }^{14} \mathrm{~N}$. We adopted two different ways to treat the evolution of ${ }^{14} \mathrm{~N}$ abundance, to find out the effect of nuclear energy production on the formation of the blue loop. We noticed that the $\mathrm{CN}$ cycle reaches the nuclear reaction equilibrium at the very beginning of the H-burning, and thus we chose $X_{\mathrm{C}}+X_{\mathrm{N}}$ as the initial value of ${ }^{14} \mathrm{~N}$ abundance. The first approach was to consider the $\mathrm{CN}$ cycle only, which meant to keep the abundance of ${ }^{14} \mathrm{~N}$ constant throughout the evolution. Models based on this approach will be called CN models 


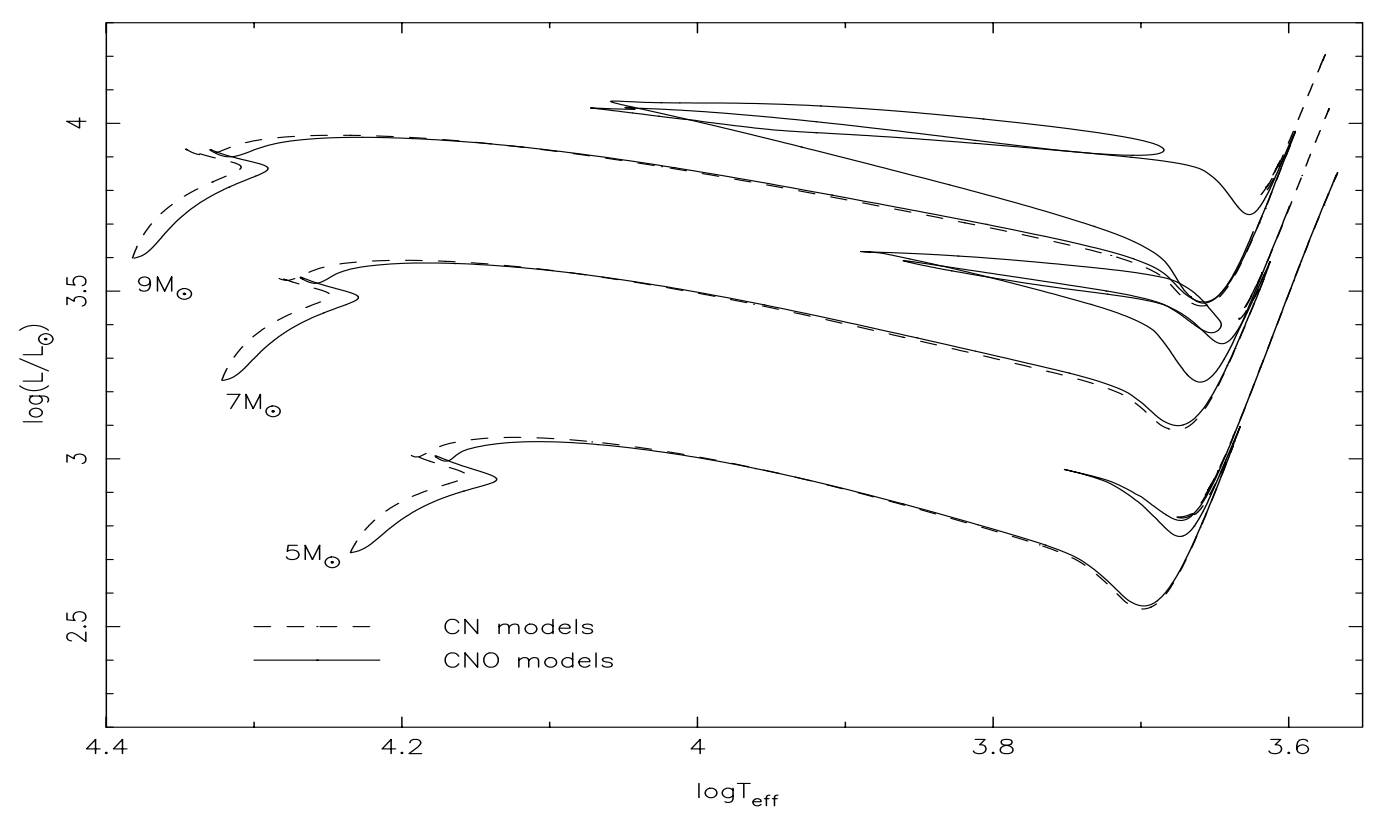

Fig. 1. The evolutionary tracks of 5,7,9 $M_{\odot}$ stars with the OPAL opacity, from the zero-age main sequence to the AGB stage. The ages when the stars become bluest in the first blue loop are $9.83 \times 10^{7}, 4.58 \times 10^{7}, 2.72 \times 10^{7}$ years respectively for the CNO models of $5,7,9 M_{\odot}$.

afterwards. The second approach dealt with the transformation of ${ }^{16} \mathrm{O}$ to ${ }^{14} \mathrm{~N}$ by an approximation introduced by Clayton (1968):

$\frac{\mathrm{dN}_{14}}{\mathrm{~d} t} \doteq-\gamma \frac{\mathrm{N}_{14}}{\tau_{14}}+\frac{\mathrm{O}_{16}}{\tau_{16}}$,

$\gamma=\frac{r_{15}^{*}}{r_{15}^{*}+r_{15}}$,

where $r_{15}^{*}$ and $r_{15}$ are the rates of reactions ${ }^{15} \mathrm{~N}(p, \gamma){ }^{16} \mathrm{O}$ and ${ }^{15} \mathrm{~N}(p, \alpha){ }^{12} \mathrm{C}$, respectively, and $\gamma$ is the branch ratio of the NO cycle that determines the fraction of $\mathrm{CNO}$ material leaking into the NO cycle. $\tau_{14}$ and $\tau_{16}$ denote the lifetimes of ${ }^{14} \mathrm{~N}$ and ${ }^{16} \mathrm{O}$ in the nuclear reactions ${ }^{14} \mathrm{~N}(p, \gamma){ }^{15} \mathrm{O}$ and ${ }^{16} \mathrm{O}(p, \gamma){ }^{17} \mathrm{~F}$ respectively. The stellar models adopting this approach are hereafter referred to as CNO models. The complete treatment of the CNO cycles is based on the nuclear-reaction network (Arnett $\&$ Truran 1969; Maeder 1983) that calculates the variation of chemical composition of all elements. Our treatment is less accurate, but runs faster and can reproduce most of the properties of the stellar models using the nuclear-reaction network. The rates of the nuclear reactions and their energy generation were taken from Caughlan \& Fowler (1988).

The OPAL opacities G91hz series (Iglesias \& Rogers 1996) were used in the high temperature region. Low temperature opacities from Alexander \& Ferguson (1994) were used to include the contribution from molecules and grains. These two sets of opacities were connected around a temperature of $8000 \mathrm{~K}$ at that fitting point they coincide very well.

The normal Population I abundance, $X=0.70, Y=0.28$, $Z=0.02$ (Anders \& Grevesse 1989; Grevesse \& Noels 1993), was adopted in our models. As a strong restriction, our results can only be applied to stars of solar-like metallicity. Within the heavy element abundance we chose $X_{\mathrm{N}}=0.0045$, which was the sum of the original abundance of ${ }^{14} \mathrm{~N}$ and ${ }^{12} \mathrm{C}$, and $X_{\mathrm{O}}=0.01$. Energy transfer by convection was treated according to the standard mixing-length theory, and the boundaries of the convection zones were determined by the Schwarzschild criterion (Cox \& Giuli 1968; Huang \& Yu 1998). The ratio of the mixing-length to local pressure scale height, $\alpha$, was chosen to be 1.8. Overshooting from the convective core was omitted. Mass loss due to the stellar wind was ignored because it is small for intermediate mass stars. Evolution models were calculated using a modified code originally described by Kippenhahn et al. (1967).

\section{Properties of evolution models}

We have calculated two series of evolution models of $5 M_{\odot}$, $7 M_{\odot}$, and $9 M_{\odot}$. These models consist of more than 1000 mass zones during the main-sequence and more than 3000 mass zones during the core He-burning phase, and evolve in about 2000 time steps from the zero-age main sequence to the early AGB stage.

Figure 1 shows the evolution tracks of the $\mathrm{CN}$ and $\mathrm{CNO}$ models. We notice that the inclusion of the NO cycle significantly expands the width of the main sequence. It can be seen that all of the CNO models show blue loops, and the extension of the blue loop increases with the stellar mass. On the opposite side, all of the $\mathrm{CN}$ models stay on the red-giant branch during the whole central He-burning phase, no matter how large the stellar mass is. Iben (1967) pointed out that the rate of reaction ${ }^{12} \mathrm{C}(\alpha, \gamma){ }^{16} \mathrm{O}$, which only influenced the energy production of the core He-burning phase, could affect the development of the blue loop. Our result differs from his, for our models deal with the energy production of the H-burning that takes place both in the main sequence and in the shell $\mathrm{H}$-burning phase.

Figure 2 shows the evolution of the abundance of ${ }^{14} \mathrm{~N}$ and ${ }^{16} \mathrm{O}$ at the center of the $7 M_{\odot}$ model during the main sequence. It can be seen that ${ }^{14} \mathrm{~N}$ quickly increases in the early stage of 


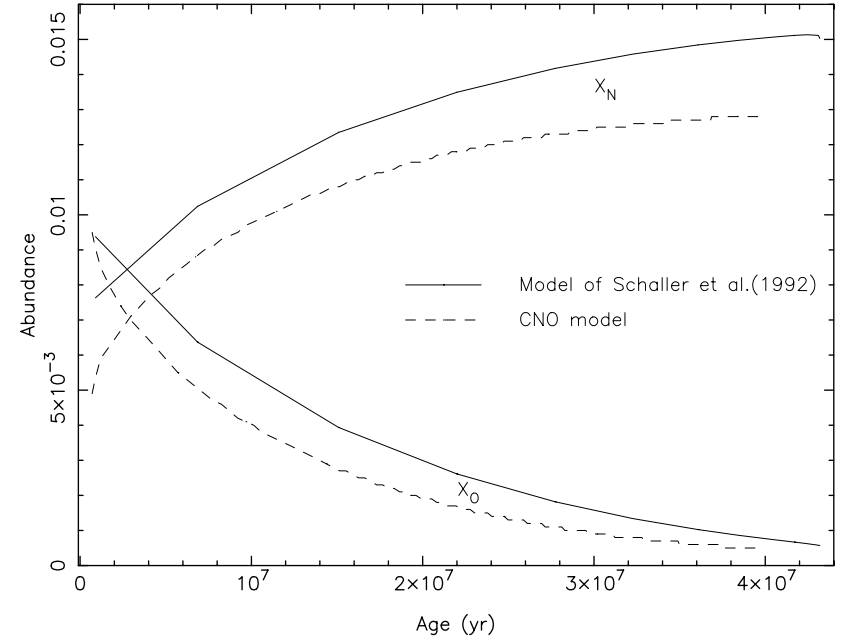

Fig. 2. The evolution of ${ }^{14} \mathrm{~N}$ and ${ }^{16} \mathrm{O}$ abundance at the center of the $7 M_{\odot}$ model in the main sequence stage. The upper two lines are for the ${ }^{14} \mathrm{~N}$ abundance, and the lower two lines for the ${ }^{16} \mathrm{O}$ abundance.

the central H-burning and approaches the nuclear balance abundance of the CNO cycles when hydrogen is almost exhausted at the center. In the majority of the lifetime of the main sequence, ${ }^{14} \mathrm{~N}$ in the $\mathrm{CNO}$ models is about twice as much as in the $\mathrm{CN}$ models. This fact explains why the CNO models are redder than the $\mathrm{CN}$ models in the main sequence on the HRD, for the CNO models need a slightly lower core temperature to produce the same amount of energy than the $\mathrm{CN}$ models do. The evolution of the abundance of ${ }^{14} \mathrm{~N}$ and ${ }^{16} \mathrm{O}$ in the models of Schaller et al. (1992), that calculated the CNO cycles by means of the nuclear reaction network, is also shown in Fig. 2. Except for small differences in the choices of the initial abundance of ${ }^{14} \mathrm{~N}$ and ${ }^{16} \mathrm{O}$, the two results are in good agreement. It is obvious that our simple approach to treat the CNO bi-cycle is accurate enough for the evolution calculations of intermediate mass stars.

The evolution tracks of the $\mathrm{CN}$ and $\mathrm{CNO}$ models converge soon after the main sequence and form almost the same RGB, but afterwards they evolve in completely different ways. Figure 3 compares in detail the evolution tracks of the $7 M_{\odot}$ models during the central He-burning phase. It can be seen that the $\mathrm{CNO}$ model is $5 \%$ brighter at point a than the $\mathrm{CN}$ model is at $\mathrm{a}^{\prime}$, which results in its envelope convection penetrating inwardly a little bit deeper than in the envelope of the $\mathrm{CN}$ model. At points $\mathrm{c}$ and $\mathrm{c}^{\prime}$, the stars fall along the RGB to reach the minimum luminosity. The luminosity of the CNO model is found to be lower than that of the CN model. Renzini et al. (1992) suggested that a star would develop a blue loop if its luminosity at this point was lower than a critical value $L_{\text {loop }}$.

During the core He-burning stage, the H-burning shell moves outward continuously in all models. As shown in Fig. 4 this thin shell, occupying only $1-5 \%$ of the total stellar mass, moves outward slowly from 0.13 to about 0.20 of the stellar mass. The movement of the H-burning shells in the CNO models gradually slows down at first, but as soon as their upper edges touch the abundance discontinuity they speed up for some time and finally slow down again when the maximum energy production layer arrives at the original abundance discontinuity. But in the $\mathrm{CN}$ models, except for a sudden jump the H-burning shells slow down monotonically with time during the whole course of moving outward. Another interesting result is that the CNO models reach the bluest tip of the first blue loop in Fig. 4 (point e) as soon as the maximum energy production layer of the H-burning shell touches the abundance discontinuity. Lauterborn et al. (1971a) discovered a similar effect before.

\section{Formation mechanism of the blue loop}

When a star develops a blue loop on the HRD, its luminosity and effective temperature increase with time before it reaches the bluest tip. Any mechanism to drive such an evolution pattern has to explain these two characteristics. It should be noticed, however, that these two characteristics deal respectively with completely different stellar properties. The stellar luminosity measures the rate of energy production in the stellar core, while the effective temperature measures how far the stellar envelope can expand for a given stellar luminosity. According to this feature of the considered problem, we investigate separately the variation of the stellar core and the stellar envelope, and place our emphasis on their mutual interactions. To show the importance of energy production in the H-burning shell, we divide a star into three regions, i.e., the shell source (including the H-burning shell), the core (interior to the H-burning shell), and the envelope (exterior to the $\mathrm{H}$ burning shell). The boundaries of the shell source are defined by the condition $\varepsilon_{\mathrm{H}}=1 \mathrm{erg} \times \mathrm{s}^{-1} \mathrm{~g}^{-1}$, where $\varepsilon_{\mathrm{H}}$ is the energy generation rate of the $\mathrm{H}$-burning. We use the $7 M_{\odot}$ models as examples for our investigations, while the results can be applied to other models.

\subsection{Global properties of the core and the envelope}

We start by investigating the global properties of the stellar core and the stellar envelope with the aid of the virial theorem:

$2 E_{\mathrm{T}}+E_{\mathrm{G}}=$ const.,

where $E_{\mathrm{T}}$ is the thermal energy and $E_{\mathrm{G}}$ is the gravitational potential of a star:

$E_{\mathrm{T}}=\frac{3}{2} \int_{M_{1}}^{M_{2}} \frac{P}{\rho} \mathrm{d} M_{r}$,

$E_{\mathrm{G}}=-\int_{M_{1}}^{M_{2}} \frac{G M_{r}}{r} \mathrm{~d} M_{r}$.

In the above equations, $P$ is the pressure, $\rho$ the density, $G$ the gravitational constant, $M_{r}$ the mass inside the sphere of radius $r$. Equation (2) is accurate if the integrals are carried out over the whole star. However, we have applied it separately to the core, the shell source, and the envelope of the $7 M_{\odot}$ models, and the results are shown in Fig. 5. It can be seen that $E_{\mathrm{G}}$ and $E_{\mathrm{T}}$ of the core vary in a similar way for both CNO and $\mathrm{CN}$ models, while those of the envelope evolve quite differently depending on whether the model develops a blue loop 


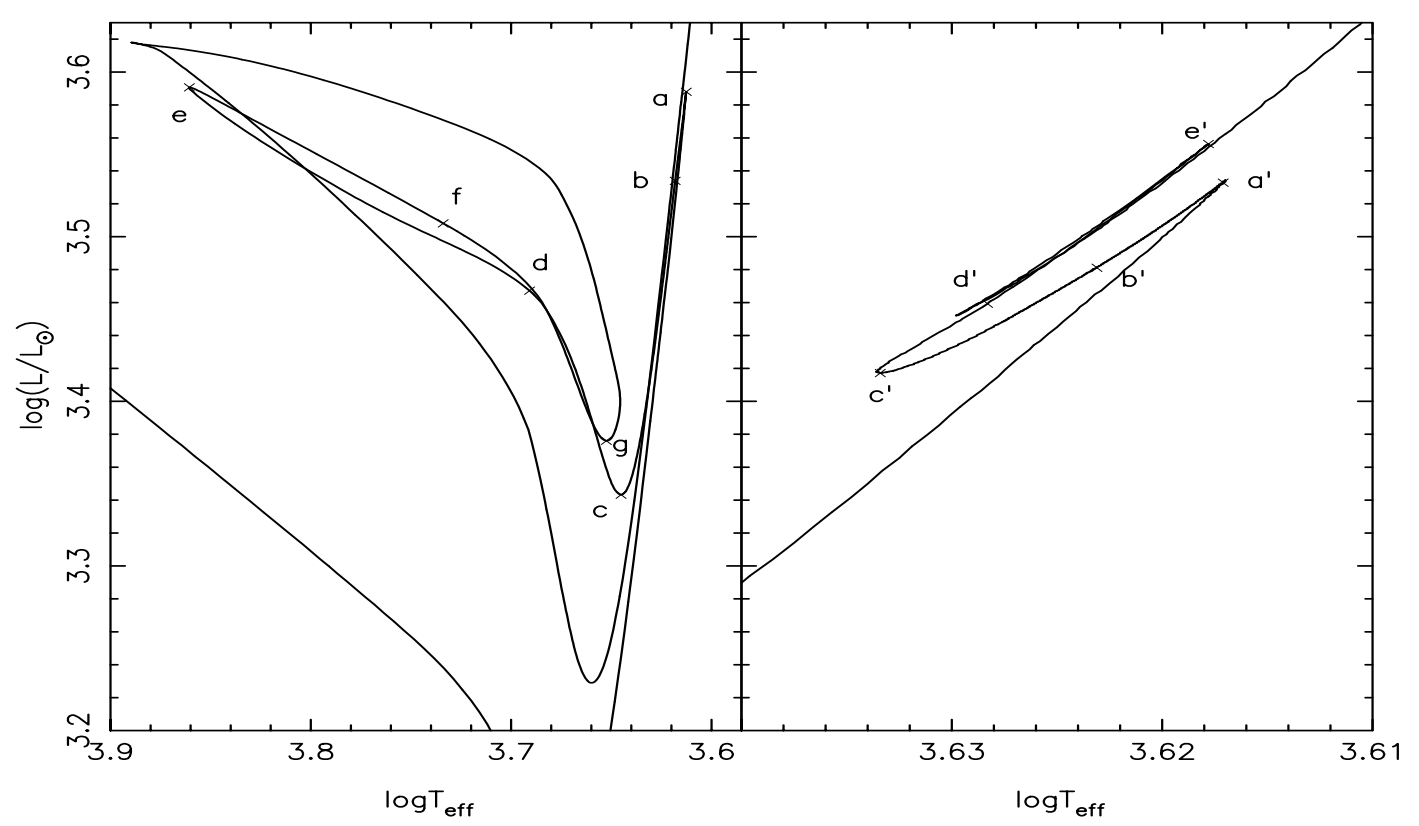

Fig. 3. Details of the evolution tracks of the $7 M_{\odot}$ models during the core He-burning stage. The left panel is for the CNO model and the right for the $\mathrm{CN}$ model. Some critical moments are marked by crosses and labelled with a-g for the $\mathrm{CNO}$ model and $\mathrm{a}^{\prime}-\mathrm{e}^{\prime}$ for the $\mathrm{CN}$ model. These labels are used in the remaining part of the paper and figures.
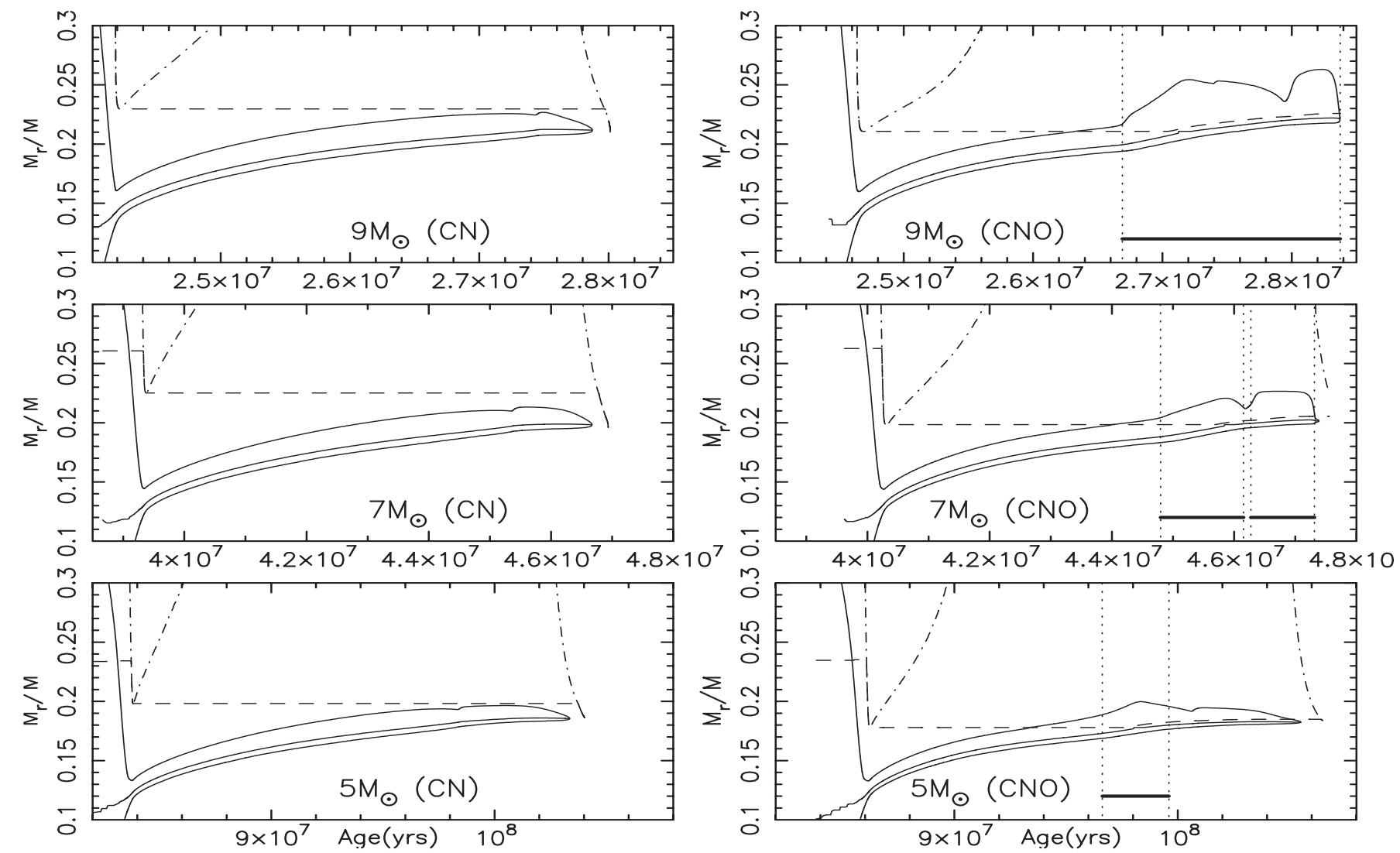

Fig. 4. Development of the envelope convection and movement of the H-burning shell in the central He-burning stage. From top to bottom in each panel are the base of the convective envelope (dot-dashed), the layer where $X=0.65$ (dashed), and the upper edge, center, and lower edge of the H-burning shell, respectively. The area between the vertical dotted lines denotes that the envelope is wholly radiative.

(the CNO model) or not (the $\mathrm{CN}$ model). This result confirms that the stellar core can barely be influenced by the stellar envelope (Hayashi et al. 1962; Fricke et al. 1971). It is evident that only the envelope responds to the blue loop in the CNO model.
It contracts at first to heat itself and then expands. An interesting result is that the core and the envelope independently obey the virial theorem to a very high accuracy (no more than about 2\%), whether the model develops a blue loop or not. This 

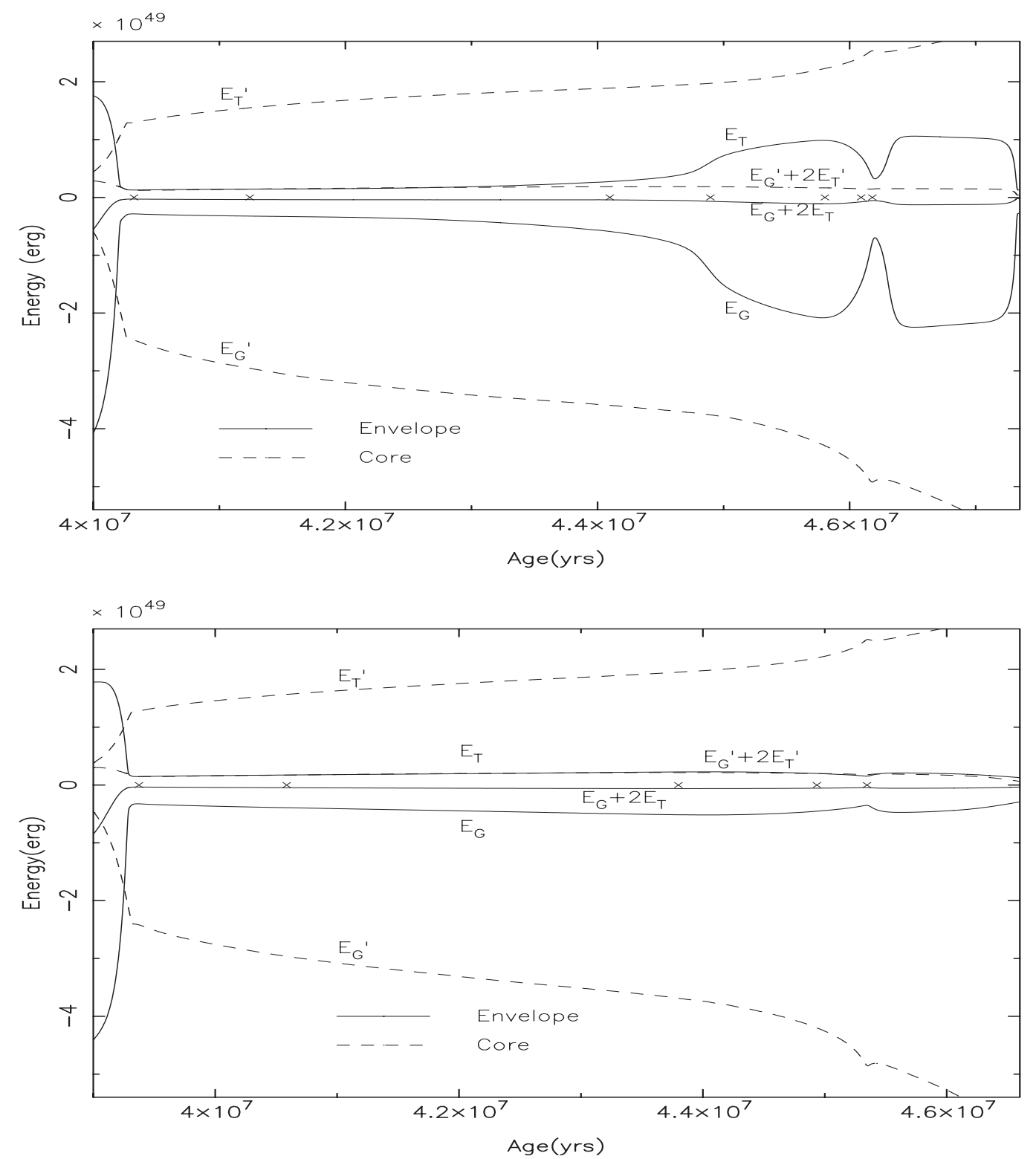

Fig. 5. Virial theorem of the core and the envelope of the $7 M_{\odot}$ models during the core He-burning phase. The upper panel is for the CNO model and the lower panel for the $\mathrm{CN}$ model.

is completely different from the case in the post main-sequence evolution, in which the core contracts while the envelope expands to form a so-called "mirror reflection" effect. The "mirror reflection" effect is a direct result of a star complying as a whole with the virial theorem (Fricke \& Strittmatter 1972).

As the H-burning shell is very thin, it does not contribute much to the total $E_{\mathrm{G}}$ and $E_{\mathrm{T}}$ as seen in Fig. 5, but the nuclear energy production from this thin shell is the main energy source to increase the luminosity of the star during the blue loop phase. What is the response of the envelope to such an increase of energy input from the bottom? The luminosity is related to the radius and effective temperature of a star by

$L=4 \pi R^{2} \sigma T_{\mathrm{eff}}^{4}$

It can be seen that the star can increase either its radius or its effective temperature to transport the extra energy supplied from its interior. However, the radius and the effective temperature cannot vary independently, since the stellar envelope obeys the virial theorem. As $T_{\text {eff }}$ roughly measures $E_{\mathrm{T}}$ and $R$ roughly measures $E_{\mathrm{G}}$ of the envelope, we obtain from Eq. (2) that increasing $R$ requires to decrease $T_{\text {eff }}$, or $T_{\text {eff }}$ increases when $R$ decreases. Thus for an increase of luminosity at the base of the envelope, the envelope will either increase its radius with decreasing the effective temperature, or increase its effective temperature with decreasing the radius. This result is an important constraint on the evolution after the star arrives at the bottom of the RGB. The star can either expand to evolve up along the RGB, or increase the effective temperature to form a blue loop. In the following subsections, we shall first investigate the reasons for the increase of the stellar luminosity, and then find out the conditions to determine which way a star will choose. 

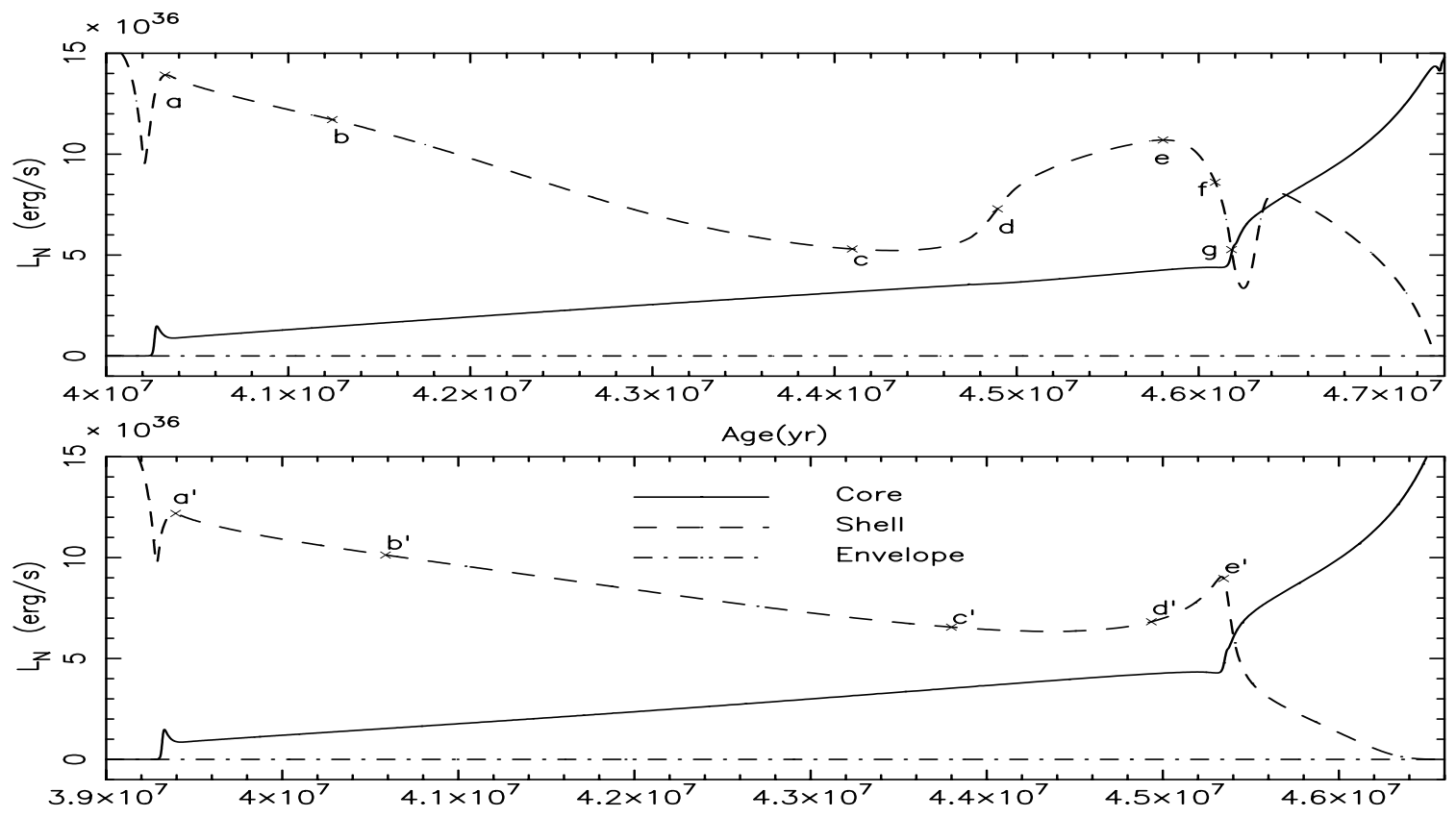

Fig. 6. The nuclear energy production in the $7 M_{\odot}$ models. The top panel is for the CNO model and the bottom panel for the CN model. The labels beside the curves correspond to the moment marked on the evolution tracks in Fig. 3.

\subsection{Nuclear energy production in the stellar core}

We have investigated the nuclear energy production by the central He-burning and the shell H-burning processes. Figure 6 shows the luminosity generated from each of the above burning regions, respectively. The evolution tracks in Fig. 3 can be understood very well with the aid of the variation of energy production in the H-burning shell. The central He-burning produces almost the same luminosity for both $\mathrm{CNO}$ and $\mathrm{CN}$ models all the time, and this result excludes the central He-burning process as a driving mechanism of the blue loop (Hayashi et al. 1962; Fricke et al. 1971). However the role of energy production from the H-burning shell is completely different. After the helium ignition in the stellar center, the H-burning luminosities reverse to increase rapidly. The H-burning shell can give more nuclear energy in the $\mathrm{CNO}$ model at point a than in the $\mathrm{CN}$ model at point $\mathrm{a}^{\prime}$, because the ${ }^{14} \mathrm{~N}$ abundance in the $\mathrm{CNO}$ model is about twice as much as in the $\mathrm{CN}$ model. Figure 7 shows the ${ }^{14} \mathrm{~N}$ abundance profile of the CNO model by the end of the main sequence. Afterwards the energy production rate of the H-burning shell slowly decreases to its minimum, and the evolution tracks drop down to the bottom of the RGB. Then the energy production rate of the H-burning shell in the CNO model increases again and reaches the second maximum, which corresponds to the evolution track going blueward and arriving at the bluest tip of the first blue loop. As the energy production in the H-burning shell excellently explains the evolution of the stellar luminosity during the blue loop, we regard it as an important factor in the driving mechanism of the blue loop. A similar effect does not appear in the $\mathrm{CN}$ model. The energy production rate in its H-burning shell decreases in most of the central He-burning phase and increases again only near the central helium exhaustion at point $\mathrm{e}^{\prime}$, and causes the star to go up again along the RGB.

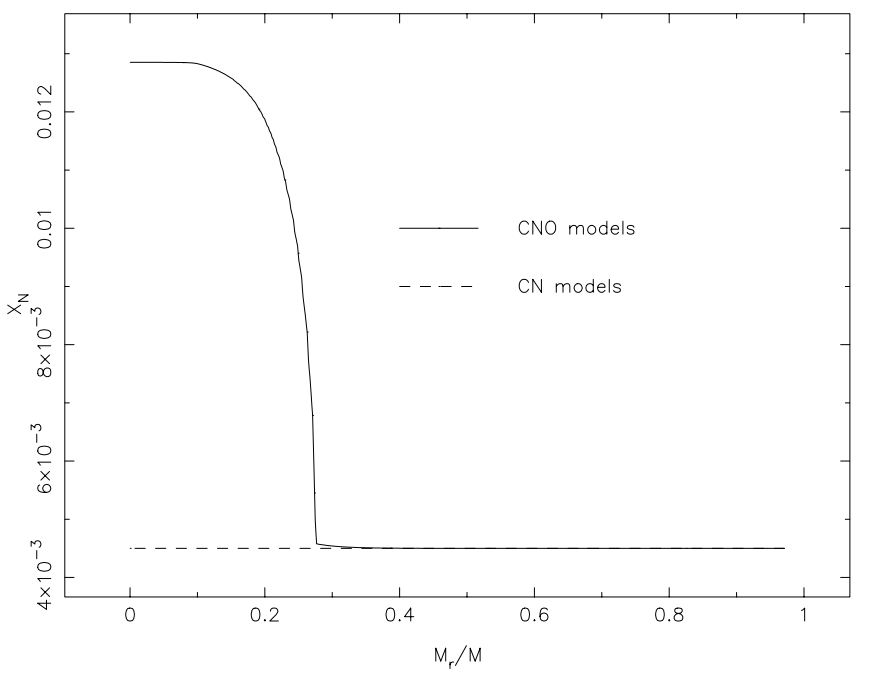

Fig. 7. Profile of ${ }^{14} \mathrm{~N}$ abundance by the end of the main sequence for the $7 M_{\odot}$ models.

What causes the increase of energy production in the H-burning shell for the CNO model? We can study this problem by separating the energy production rate, $\varepsilon_{\mathrm{H}}$, into several ingredients:

$\frac{\Delta \varepsilon_{\mathrm{H}}}{\varepsilon_{\mathrm{H}}} \approx \frac{\Delta X}{X}+\frac{\Delta X_{\mathrm{N}}}{X_{\mathrm{N}}}+\frac{\Delta \rho}{\rho}+n \frac{\Delta T}{T}$,

where $n$ is an index to measure the temperature dependence of the H-burning rate. Figure 8 shows the variation rate of each factor at the layer $\varepsilon_{\mathrm{H}}=$ maximum. After the onset of the central He-burning, the increasing ${ }^{12} \mathrm{C}$ abundance accelerates the central He-burning through the reaction ${ }^{12} \mathrm{C}(\alpha, \gamma){ }^{16} \mathrm{O}$ (Renzini et al. 1992), and the excessive release of energy therefrom will increase the central temperature. The increasing central 

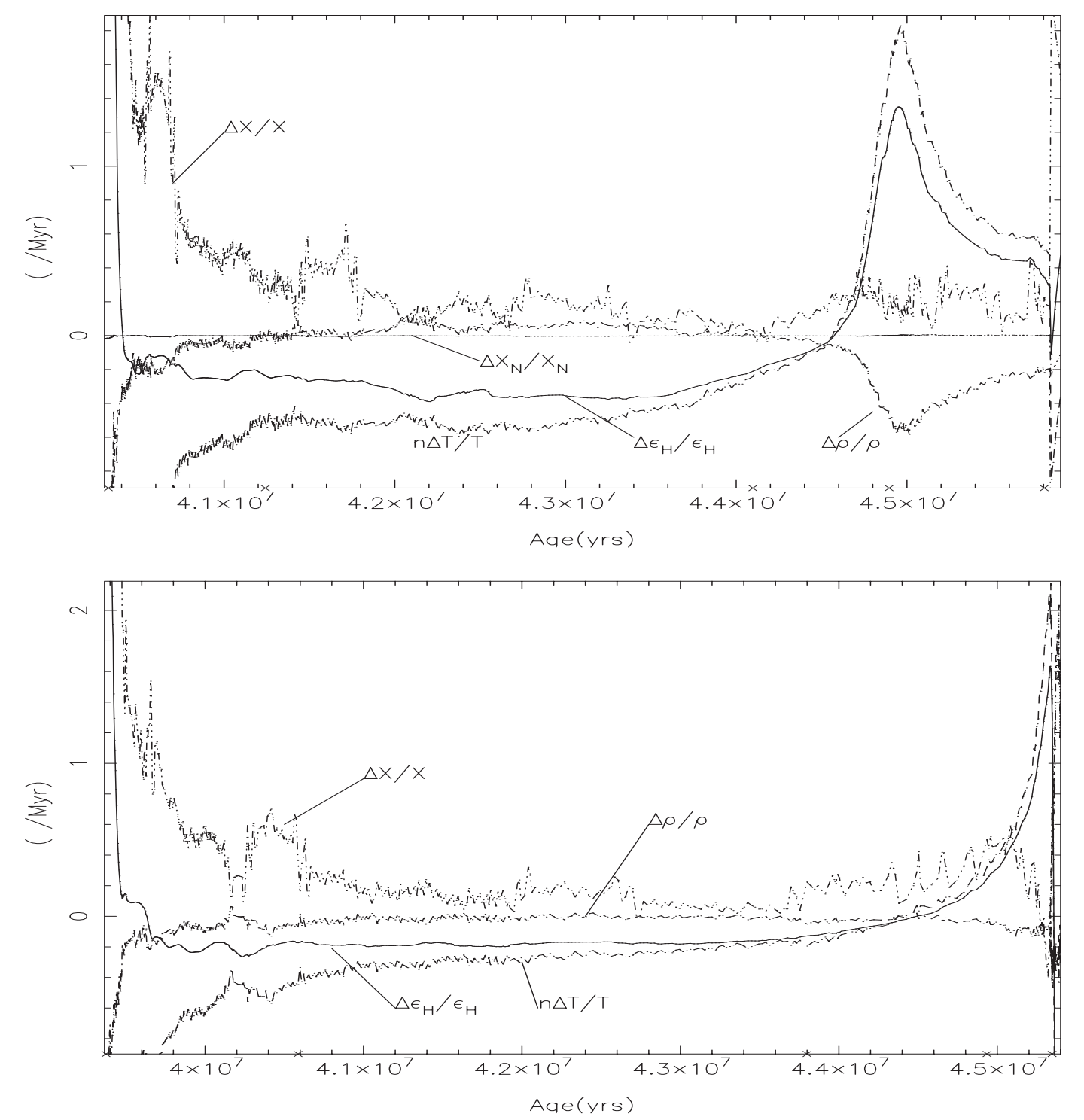

Fig. 8. The variation rates of the physical ingredients that determine the nuclear energy production at the layer $\varepsilon_{\mathrm{H}}=$ maximum in the $7 M_{\odot}$ models. The top panel is for the CNO model and the bottom for the $\mathrm{CN}$ model.

temperature interacts with the H-burning shell in two ways. Firstly, the H-burning shell will be heated by the core, because there is a radiative region between the He-burning core and the H-burning shell. We call this effect "T-prompt", which drives the temperature of the H-burning shell synchronously increasing to transfer more and more heat. This effect can be easily recognized in Fig. 8. The temperature variation rate always goes up except for the last short period just before the central He-burning stops. Secondly, the increasing central temperature induces the expansion of the stellar core. We refer to this as the "push effect", i.e., it pushes the H-burning shell to a lower temperature region and results in the decrease of the H-burning luminosity. Figure 9 reveals the departure of the H-burning shell from the stellar center. It can be seen in Fig. 8 that all quantities on the right hand side of Eq. (6) vary quickly at first and then gradually slow down as the H-burning shell departs farther and farther from the stellar core. It is clear that the nearer the H-burning shell is initially located from the helium core, the stronger the "push effect" will be. These two effects exist simultaneously as two opposite constraints on the efficiency of the shell energy production. During the early phase of the central He-burning, the "push effect" overwhelms the "T-prompt" and the H-burning luminosity decreases. But with the departure of the H-burning shell from the stellar core, the "push effect" gradually reduces and its effect can be largely compensated by the "T-prompt". Inside the H-burning shell, however, hydrogen is exhausted at the bottom while supplied with higher and higher abundance from the top along the chemical profile. This makes the H-burning more and more efficient and will be referred to as "X-prompt" hereafter.

In the case of the CNO model, the "push effect" is strong enough during the early stage of the core He-burning (from a to c), resulting in rapid decrease of temperature and energy production in the shell source seen in Fig. 8. Correspondingly, the whole envelope slowly contracts and is heated at the same time. With the help of "T-prompt" and "X-prompt" effects, especially after the chemical discontinuity moves into the $\mathrm{H}$ burning shell, more and more energy is generated to cause the temperature increasing in the H-burning shell. The $\mathrm{CN}$ model shares similar properties during the early stage of the core 
He-burning (from $\mathrm{a}^{\prime}$ to $\mathrm{c}^{\prime}$ ), but the decrease of the energy production and temperature lasts much longer until the envelope falls down to increase the temperature and energy production of the H-burning shell just before helium is almost exhausted in the stellar core. Our numerical results support the idea that the hydrogen discontinuity on the chemical profile takes effect on the formation of the blue loop (Robertson 1972; Stothers \& Chin 1973; Schlesinger 1977; Stothers \& Chin 1991a). Comparing the CNO and CN models, we find that the decrease of temperature in the CNO model in the early stage of the central He-burning is stronger but shorter than in the $\mathrm{CN}$ model. The reason is due to the higher ${ }^{14} \mathrm{~N}$ abundance in the H-burning shell that causes faster depletion of hydrogen and faster outward movement of the shell source in the CNO model, i.e., a stronger "push effect".

\subsection{Development of convection in the stellar envelope}

The stellar envelope adjusting itself from convective to radiative was considered by Chiosi et al. (1992) as the cause of the blue loop. We investigate this process by defining an envelope convective ratio:

$\eta=\frac{M_{\text {con }}}{M_{\text {env }}}$

to estimate the development of convection in the stellar envelope. $M_{\mathrm{env}}$ and $M_{\mathrm{con}}$ are the total mass of the envelope and the mass of the convection zones within the envelope, respectively. The energy transportation in the stellar envelope is connected strongly with the development of convection. The more the convective motion penetrates downwards (larger $\eta$ ), the more the energy is transferred and the envelope structure is determined by convection. Figure 10 shows the evolution of the envelope convective ratio for the $\mathrm{CNO}$ and $\mathrm{CN}$ models of $7 M_{\odot}$. We see that both $\mathrm{CNO}$ and $\mathrm{CN}$ models have very thick convective envelopes at the top of the RGB, that occupy more than $90 \%$ of the envelope mass. As the stars evolve down along the RGB, the convection in the CNO model quickly recedes and nearly disappears shortly after the star begins the blue loop evolution in Fig. 3. At the critical point $\mathrm{c}$ where the star has been down to the bottom of the RGB, $\eta$ decreases to 0.32 and the envelope becomes radiation-dominated. In the envelope of the $\mathrm{CN}$ model, however, more than half of the envelope is always convective, and the envelope is always convection-dominated.

For an increase of luminosity at the base of the stellar envelope, the envelope will respond to this variation in two ways according to the physical structure of the envelope. For a completely radiative envelope $(\eta \approx 0)$, the temperature will be increased to enlarge the radiative conductivity efficiency, and the star will evolve blueward. For example, a star with a larger mass and thus a higher luminosity will have a higher effective temperature on the main sequence. For a completely convective envelope $(\eta \approx 1)$, on the other hand, larger heat input will drive stronger convective motion and the convection zone extending larger, which leads to a smaller effective temperature and redward evolution. Stars evolving along the RGB belong to this case. Therefore for a mixed case that there are both radiative and convective regions in a stellar envelope, there should be a
Table 1. Properties of evolution models on the RGB.

\begin{tabular}{llcccc}
\hline \hline Mass & Model & $\lg \left(L_{\mathrm{a}} / L_{\odot}\right)$ & $\eta_{\mathrm{a}}$ & $\lg \left(L_{\mathrm{c}} / L_{\odot}\right)$ & $\eta_{\mathrm{c}}$ \\
\hline $9 M_{\odot}$ & $\mathrm{CNO}$ & 3.976 & 0.942 & 3.728 & 0.414 \\
$9 M_{\odot}$ & $\mathrm{CN}$ & 3.935 & 0.920 & 3.780 & 0.539 \\
$7 M_{\odot}$ & $\mathrm{CNO}$ & 3.588 & 0.939 & 3.343 & 0.321 \\
$7 M_{\odot}$ & $\mathrm{CN}$ & 3.536 & 0.907 & 3.417 & 0.528 \\
$5 M_{\odot}$ & $\mathrm{CNO}$ & 3.096 & 0.949 & 2.769 & 0.241 \\
$5 M_{\odot}$ & $\mathrm{CN}$ & 3.039 & 0.925 & 2.834 & 0.371 \\
\hline
\end{tabular}

critical value of $\eta$, and above it the star evolves redward and below it the star evolves blueward. From Fig. 10 we find that the critical value, $\eta_{\text {crit }}$, is somewhere between 0.32 and 0.53 for the $7 M_{\odot}$ star. Renzini et al. (1992) reached a similar result. They found that a blue loop would be developed if the stellar luminosity was lower than a critical value $L_{\text {loop }}$ at the bottom of the RGB. The luminosity and the envelope convective ratio are closely related to each other at the bottom of the RGB, and a lower $L$ will leads to a smaller $\eta$. We regard a lower $\eta$ as a clear indication of underdevelopment of convection in the stellar envelope, and consider this as an important condition for a star to develop a blue loop. However, Renzini et al. (1992) considered a lower $L$ to satisfy the driving condition of the modified gravothermal hysteresis cycle and to form a blue loop. As $L_{\text {loop }}$ depends much more sensitively on the stellar mass than $\eta_{\text {crit }}$ does, $\eta_{\text {crit }}$ can be taken as a more convenient criterion and applied to wide range of stellar masses.

Table 1 summaries the similar properties of the other considered models. The luminosity and $\eta$ are given in the third and fourth column for the models at the top of the RGB while in the fifth and sixth column for those at the bottom of the RGB. It can be found in Table 1 that the values of $\eta$ at point $\mathrm{c}$ and $\mathrm{c}^{\prime}$ increase with the stellar mass, and the values in the $\mathrm{CN}$ models are always higher than in the CNO models. If we take the average of the $\mathrm{CN}$ and $\mathrm{CNO}$ models as an approximation to $\eta_{\text {crit }}$, we find that $\eta_{\text {crit }}$ has a weak dependence on the stellar mass. It can be found that $\eta_{\text {crit }}$ increases with the stellar mass, which implies that stars with higher masses are more suitable to form blue loops.

\subsection{Discussions on some factors of the blue loop}

Based on the previous analyses, we have identified the conditions to form a blue loop during the central He-burning stage. The enhancement of the energy production rate in the H-burning shell due to the hydrogen discontinuity moving into the shell source results in the increase of the stellar luminosity, while the underdevelopment of convection in the stellar envelope makes the envelope contract and the star evolves blueward to respond such an increase of luminosity at the base of the stellar envelope. In our investigations, the CNO models satisfy these conditions and develop extensive blue loops, while the $\mathrm{CN}$ models have very thick convection zone in the envelope and evolve redward. Higher ${ }^{14} \mathrm{~N}$ abundance in the H-burning shell plays a critical role in the formation of the blue loop for the CNO models. It helps the CNO models have brighter luminosity at the top of the RGB and leave the chemical 


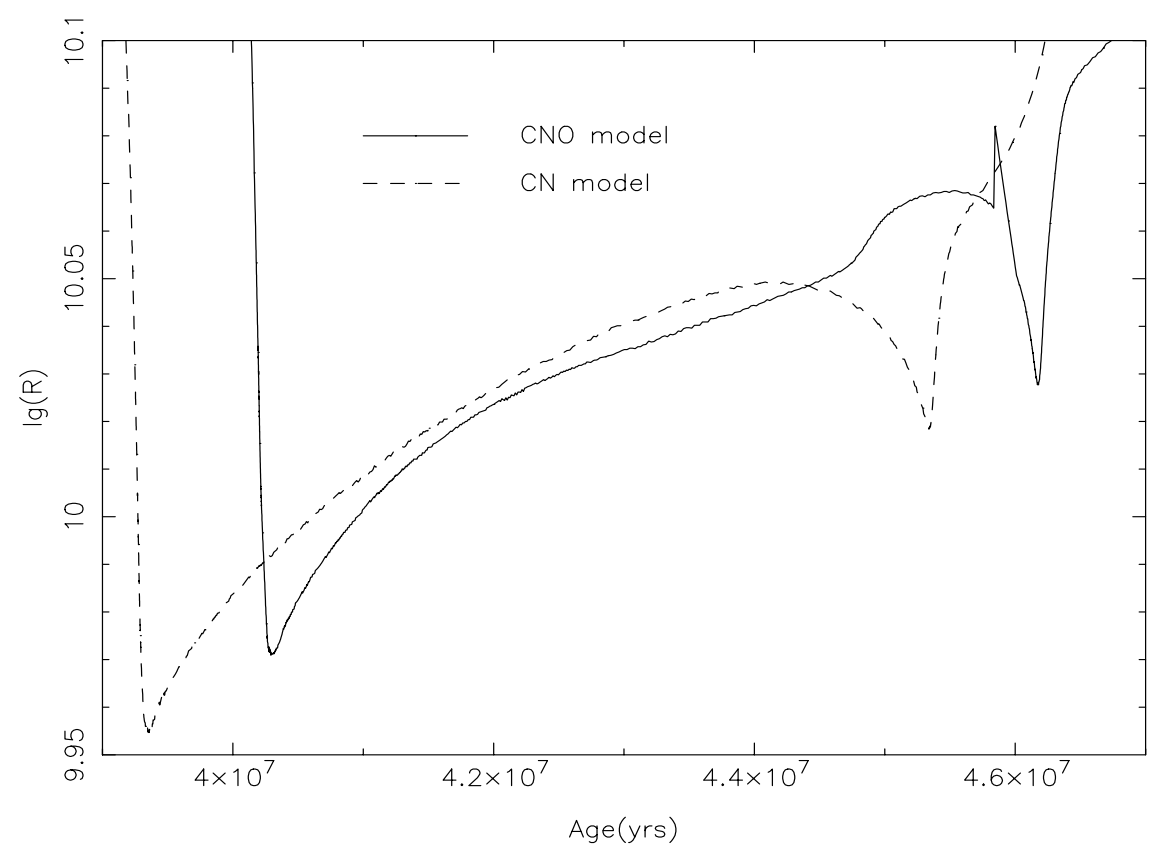

Fig. 9. The location of the center of the H-burning shell for the $7 M_{\odot}$ models.
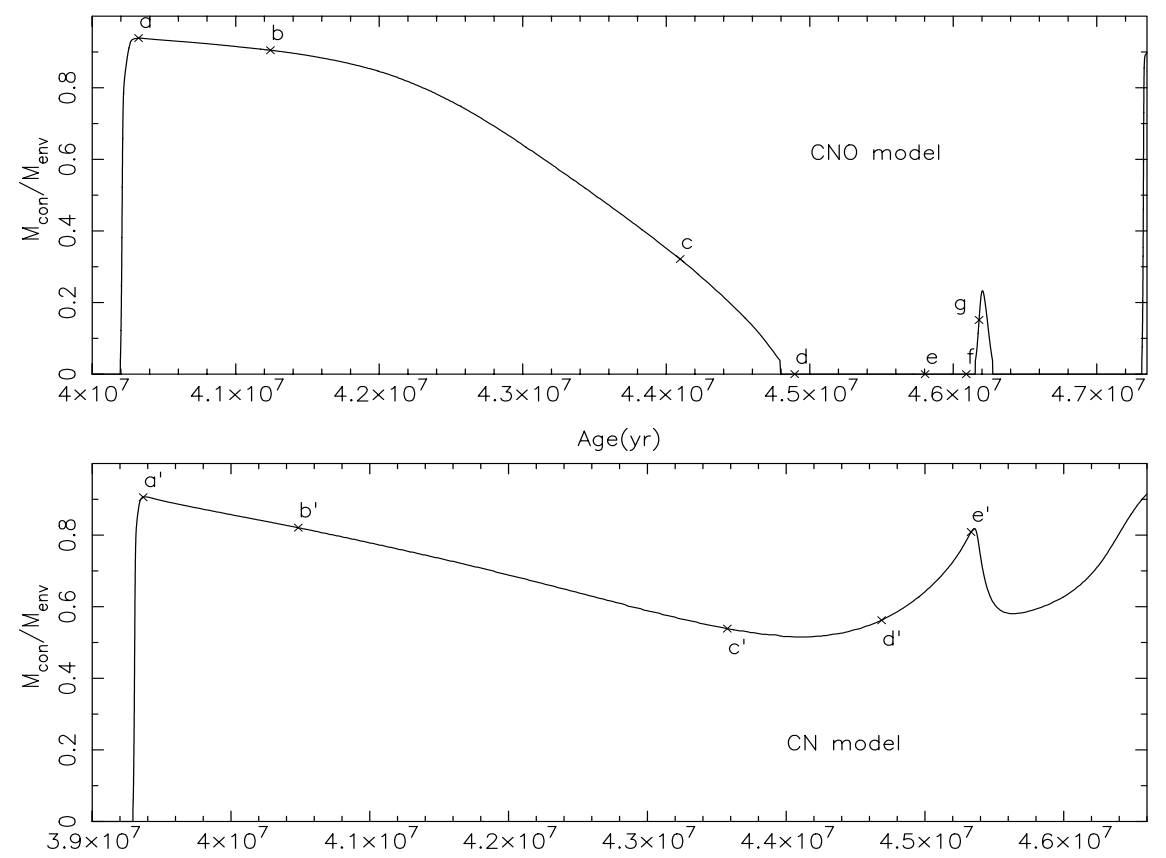

Fig. 10. Evolution of the envelope convective ratio for the $7 M_{\odot}$ models. The top panel is for the CNO model and the bottom for the $\mathrm{CN}$ model.

discontinuity closer to the stellar core, which favors the subsequent "X-Prompt" to enhance the energy production rate of the H-burning shell. The higher ${ }^{14} \mathrm{~N}$ abundance also leads to a stronger "push effect" as discussed before, which quickly reduces the energy production in the H-burning shell in the preloop phase and results in a smaller $\eta$ value at the bottom of the RGB.

The tendency to become bluer during the blue loop phase is not unlimited, and two processes can prevent the blueward evolution. Firstly, helium exhaustion in the stellar core can terminate the blue loop, as pointed out by Lauterborn et al. (1971a) and Fricke et al. (1971). Secondly, when the center of the H-burning shell touches the abundance discontinuity in our CNO models as shown in Fig. 3, the "X-Prompt" no longer works and the energy production in the H-burning shell begins to decrease. Then the reverse process sets in and the star moves back to the red region.

Now we discuss the effects of some factors of the blue loop based on our present theory. First we consider the convective overshooting that is well known to have significant influence on the blue loop. Overshooting from the convective core can bring more H-rich material into the nuclear burning core in the main sequence and enlarge the effective nuclear burning region. This effect results in the chemical profile displacing farther 
from the stellar core, as well as the abundance discontinuity formed later by the downward penetration of the envelope convection. In the subsequent evolution, the "push effect" becomes weaker and the "X-prompt" takes effect later, which makes the blue loop more difficult to form (Matraka et al. 1982; Huang \& Weigert 1983; Bertelli et al. 1985). However, the downward overshooting from the base of the convective envelope can prompt the blue loop evolution (Alongi et al. 1991; Stothers \& Chin 1991a), because it makes the hydrogen discontinuity on the chemical profile nearer to the shell source, so that the " $\mathrm{X}$ prompt" can take effect earlier. Downward overshooting brings also helium to the stellar envelope and decreases the envelope opacity, which is another effect to depress the development of convection and to promote the blue loop evolution.

Mass loss through the stellar wind, by throwing away some material from the stellar surface, makes the chemical profile be located in the lower temperature region and therefore suppresses the blue loop (Bertelli et al. 1985; Renzini et al. 1992). But great stellar wind of massive stars can strip off a considerable part of the stellar envelope and the stars evolve to the blue supergiant by greatly reducing the radius (Renzini et al. 1992; Salasnich et al. 1999). As it is quite different from what we are interested in the present paper, we shall not go into detail on this problem.

Opacity, which is an important ingredient in the stellar structure, can certainly influence the blue loop evolution (Stothers \& Chin 1994), but it acts in a different way from those factors discussed above. It is found that the OPAL opacity is greater than the LAOL opacity in some temperature regions, so that radiation cannot transfer energy as efficiently in a stellar envelope based on the OPAL opacity as in one based on the LAOL opacity. Sometimes we find an additional convection zone located where the opacity enhancement takes place. As a result, a stellar envelope based on the OPAL opacity usually has a larger $\eta$ than that based on the LAOL opacity, and thus it is more difficult to form blue loops (Li 1998).

The metallicity influences the blue loop evolution in two aspects. Firstly, the abundance of ${ }^{12} \mathrm{C},{ }^{14} \mathrm{~N}$, and ${ }^{16} \mathrm{O}$ determines the energy production of the CNO cycles. Secondly, $Z$ is sensitively related to opacity. Models of intermediate mass stars with very low metallicity may experience extensive blue loop evolution (Alcock \& Paczynski 1978; Castellani et al. 1990; Dominguez et al. 1999). We shall investigate this problem in a separate paper.

\section{Discussions and conclusions}

In the present paper we have investigated the effect of the CNO cycles on the formation of the blue loop. We have adopted two ways to treat the $\mathrm{CNO}$ cycles. The $\mathrm{CN}$ models only consider the $\mathrm{CN}$ cycle by keeping the ${ }^{14} \mathrm{~N}$ abundance constant in the whole evolution, while the $\mathrm{CNO}$ models include the NO cycle in addition in an approximate approach to allow ${ }^{16} \mathrm{O}$ to be gradually transformed into ${ }^{14} \mathrm{~N}$. A significant result is that all CNO models develop extensive blue loops but all $\mathrm{CN}$ models do not.

We have explored the triggering of the blue loop through careful comparisons of the properties of the $\mathrm{CN}$ and $\mathrm{CNO}$ models. We have noticed an important feature of the blue loop, i.e., the increase of luminosity is determined by the nuclear energy production in the stellar core while the increase of effective temperature measures how far the stellar envelope can expand for a given luminosity at its base. Accordingly, we have separately investigated the physical structure in both stellar cores and stellar envelopes, placing our emphasis on the response of the stellar envelope to an increase of luminosity at its base.

It has been found that the stellar envelope obeys the virial theorem to a very high accuracy. This makes the blue loop evolution basically different from the rapid movement of the star from blue to red on the HRD just after the main sequence. According to the virial theorem, the star expands when it becomes cooler (evolving along the RGB), or it contracts when it becomes hotter (evolving as a blue loop). Thus in a convectiondominated envelope, extra heat from its base will lead to more developed convective motion and the decrease of the effective temperature, and the star evolves up along the RGB. However in a radiation-dominated envelope, the increase of luminosity requires the increase of the envelope temperature to enlarge the radiation transfer efficiency, and the star develops a blue loop. We have introduced an envelope convective ratio $\eta$, which is defined as the envelope convection mass divided by the total envelope mass, to measure the development of convection in the stellar envelope. It is clear that there must be a critical ratio, $\eta_{\text {crit }}$, to determine the formation of a blue loop if $\eta<\eta_{\text {crit }}$. It has been found that the critical ratio $\eta_{\text {crit }}$ is between 0.3 and 0.45 for the considered models, and it shows a weak dependence on the stellar mass.

It has been found that the enhancement of energy production in the H-burning shell is the main cause for the increase of the stellar luminosity. The movement of the H-burning shell along the chemical profile is essential for the increase of the energy production rate, especially after the hydrogen discontinuity moves into the H-burning shell (Lauterborn et al. 1971b; Robertson 1972; Stothers \& Chin 1973; Schlesinger 1977; Stothers \& Chin 1991a). We have found that the higher ${ }^{14} \mathrm{~N}$ abundance in the H-burning shell plays a crucial role in the formation of the blue loop for the CNO models. It helps the CNO models to have brighter luminosity at the top of the RGB and to leave the chemical discontinuity closer to the shell source, which favors the subsequent "X-Prompt" to enhance the energy production rate of the H-burning shell. The higher ${ }^{14} \mathrm{~N}$ abundance also makes the shell source deplete hydrogen so efficiently in the pre-loop phase that the energy production rate of the H-burning shell decreases rapidly to get $\eta$ lower than the critical value.

We have discussed some factors of the blue loop based on our new theory. Overshooting from the convective core makes the chemical profile displace farther from the stellar core, as well as the abundance discontinuity formed later by downward penetration of the envelope convection. In the subsequent evolution, the "X-prompt" takes effect later, which makes the blue loop more difficult to form. The opacity enhancement of the OPAL over the LAOL leads to stronger convective motion and larger $\eta$ in the stellar envelope, and thus makes the blue loop more difficult to occur. However the present theory can only be applied to stars of solar-like metallicity. We shall 
investigate the effects of metallicity on the formation of the blue loop in a subsequent paper.

Acknowledgements. This work is co-sponsored by the NSFC of China through project number 19833040 and 10173024, National Key Fundamental Research Project through grant G1999075405 and G2000078401. Fruitful discussions with R. Q. Huang, S. L. Bi, J. Y. Yang, J. H. Guo, and Y. R. Zeng are highly appreciated. Special thanks go to an anonymous referee whose suggestions helped to clarify some important points.

\section{References}

Alcock, C., \& Paczynski, B. 1978, ApJ, 223, 244

Alexander, D. R., \& Ferguson, J. W. 1994, ApJ, 437, 879

Alongi, M., Bertelli, G., Bressan, A., \& Chiosi, C. 1991, A\&A, 244, 95

Anders, E., \& Grevesse, N. 1989, Geochim. Cosmochim. Acta, 53, 197

Arnett, W. D., \& Truran, J. W. 1969, ApJ, 157, 339

Bertelli, G., Bressan, A. G., \& Chiosi, C. 1985, A\&A, 150, 33

Bressan, A., Fagotto, F., Bertelli, G., \& Chiosi, C. 1993, A\&AS, 100, 647

Brunish, W. M., \& Becker, S. A. 1990, ApJ, 351, 258

Bono, G., Caputo, F., Cassisi, S., et al. 2000, ApJ, 543, 955

Carson, T. R., \& Stothers, R. 1976, ApJ, 204, 461

Cassisi, S., Castellani, V., Salaris, M., \& Straniero, O. 1994, A\&A, 282,760

Castellani, V., Chieffi, A., \& Straniero, O. 1990, ApJS, 74, 463

Caughlan, G. R., \& Fowler, W. A. 1988, Atomic Data and Nuclear Data Tables, 40, 283

Chiosi, C., \& Maeder, A. 1986, ARA\&A, 24, 329

Chiosi, C., Bertelli, G., \& Bressan, A. G. 1992, ARA\&A, 30, 235

Clayton, D. D. 1968, Principles of Stellar Evolution and Nucleosynthesis (U.S.: McGraw-Hill Inc.)

Cox, J. P., \& Giuli, R. T. 1968, Principles of Stellar Structure (New York: Gordon and Breach)

Dominguez, I., Chieffi, A., Limongi, M., \& Straniero, O. 1999, ApJ, 524,226

El Eid, M. F. 1995, MNRAS, 275, 983

Endal, A. S., \& Sofia, S. 1976, ApJ, 210, 184

Evans, N. R. 1993, AJ, 105, 1956

Fujimoto, M. Y., \& Iben, I. Jr. 1991, ApJ, 374, 631

Fricke, K. J., Stobie, R. D., \& Strittmatter, P. A. 1971, MNRAS, 154, 23
Fricke, K. J., \& Strittmatter, P. A. 1972, MNRAS, 156, 129

Grevesse, N., \& Noels, A. 1993, in Origin and Evolution of the Elements, ed. N. Prantzos, E. Vangioni, \& M. Cassé (Cambridge: Cambridge Univ. Press), 15

Hayashi, C., Hoshi, R., \& Sugimoto, D. 1962, Progr. Theor. Phys. Suppl. (Kyoto), No. 22

Hofmeister, E., Kippenhahn, R., \& Weigert, A. 1964, Z. Astrophys., 60,57

Huang, R. Q., \& Weigert, A. 1983, A\&A, 127, 309

Huang, R. Q., \& Yu, K. N. 1998, Stellar Astrophysics, (Singapore: Springer-Verlag)

Iben, I. Jr. 1967, ARA\&A, 5, 571

Iben, I. Jr. 1993, ApJ, 415, 767

Iben, I. Jr., \& Renzini, A. 1984, Phys. Rep., 105, 329

Iglesias, C. A., \& Rogers, F. J. 1996, ApJ, 464, 943

Kippenhahn, R., Thomas, H. C., \& Weigert, A. 1965, Z. Astrophys., 61,241

Kippenhahn, R., \& Weigert, A. 1967, Z. Astrophys., 65, 251

Kippenhahn, R., Weigert, A., \& Hofmeister, E. 1967, Methods in Computational Physics, 7 (New York: Academic Press), 129

Kippenhahn, R., Meyer-Hofmeister, E., \& Thomas, H. C. 1970, A\&A, 5,155

Kippenhahn, R., \& Weigert, A. 1990, Stellar Structure and Evolution (Berlin: Springer-Verlag)

Lauterborn, D., Refsdal, S., \& Weigert, A. 1971a, A\&A, 10, 97

Lauterborn, D., Refsdal, S., \& Roth, M. L. 1971b, A\&A, 13, 119

Lauterborn, D., \& Siquig, R. A. 1974, ApJ, 191, 589

Li, Y. 1998, Progress in Astronomy, 16, 92

Lynden-Bell, D., \& Wood, R. 1968, MNRAS, 138, 495

Maeder, A. 1983, A\&A, 128, 113

Matraka, B., Wassermann, C., \& Weigert, A. 1982, A\&A, 107, 283

Mermilliod, J. C. 1981, A\&AS, 44, 467

Renzini, A., Greggio, L., \& Ritossa, C. 1992, ApJ, 400, 280

Robertson, J. W. 1972, ApJ, 173, 631

Salasnich, B., Bressan, A., \& Chiosi, C. 1999, A\&A, 342131

Schaller., G., Schaerer, D., Meynet, G., \& Maeder, A. 1992, A\&AS, 96, 269

Schlesinger, B. M. 1977, ApJ, 212, 507

Schmidt, E. G. 1984, ApJ, 287, 261

Stothers, R. B., \& Chin, C. W. 1968, ApJ, 152, 225

Stothers, R. B., \& Chin, C. W. 1973, ApJ, 175, 555

Stothers, R. B., \& Chin, C. W. 1991a, ApJ, 374, 288

Stothers, R. B., \& Chin, C. W. 1991b, ApJ, 381, L67

Stothers, R. B., \& Chin, C. W. 1993, ApJ, 412, 294

Stothers, R. B., \& Chin, C. W. 1994, ApJ, 421, L91 